\title{
BROUGHT ABOUT BY THE SUSTAINABLE DEVELOPMENT GEOMATICS IN THE ERA OF BIG DATA OPPORTUNITIES AND CHALLENGES
}

\author{
Meini Li, Xiaosheng Liu, Jianfeng Ao \\ School of Architectural and Surveying Engineering, Jiangxi University of Science and Technology, \\ Ganzhou341000, PR China
}

KEY WORDS: Big Data, Sustainable Development, Geomatics, Opportunities, Challenges

\begin{abstract}
:
Under the background in the era of big data, in order to promote sustainable development of the geomatics for a long time, this paper studies the opportunities and challenges faced by current geographic information science through quantitative analysis, literature research and case analysis, such as favorable growth environment, the growing demand for services, the transformation of traditional management concepts; the ability to manage and control virtualized data, the security protection and proper use of massive data, and the improvement of relevant laws and regulations. Finally, in order to adapt to the development of the era of big data, it puts forward new requirements for the diversification and service mode of geomatics, promotes the all-round development of geomatics, and provides more refined and personalized services for the majority of users.
\end{abstract}

\section{Introduction}

"Big data" has become another technological reform boom that has emerged after the Internet of things and cloud computing. This concept was put forward by McKinsey (Chunjin Tan,2017) company, which makes the whole people in the new information age of data prosperity, data explosion and digital earth It aims to conduct mining and analysis of massive data, obtain new rules among original data, acquire new information and create new value.

The emerging disciplines and techniques for mapping and mapping geographic information have been proposed since 1992 and are rapidly booming(Bojie Fu,2014). In the context of the era of big data, this discipline and technology conform to the development of the times, meet the needs of various fields at different depths, and constantly present new concepts, new ideas and new methods in academic theory and methodology(Fuhua Ren,2015), prompting its healthy and sustainable development. Based on this, this paper studies the opportunities and challenges brought about by the sustainable development of the industry in the era of big data.

\section{Opportunities for geomatics in the era of big} data

Big data has been rapidly expanding from the IT industry to other fields(Lili Cui et al,2017), which has far-reaching impact on every industry, and the geomatics industry is the most prominent. The high attention of the central department, to the growing demand for its application in the social field, and the real-time update and improvement of related professional levels provide a new environment, new market and new guarantee for the growth of the industry(Lazer D et al, 2014), and meanwhile provide more intelligent services for the public in an all-round way. At present, the development opportunities brought by it are mainly reflected in the following aspects:

Corresponding Author. Present address: School of Architectural and Surveying Engineering, Jiangxi University of Science and Technology,Ganzhou341000,PR China. Tel:86+15802951640

E-mail address:1606440893@qq.com 
2.1 The country attaches great importance to the development opportunities brought by it

The explicit guidance of the Outline of "the 13th Five-Year Plan for Surveying and Mapping Geographic Information Industry"(Rabari C et al, 2015)has pointed out the forward goals for the sustainable development of the geographic information industry and vigorously optimized the development environment. The state stands at a global level, it puts forward new requirements for its development, and the economic aspect makes it provide a basic guarantee for the superstructure. In the aspect of deepening the reform, we should establish a diversified investment mechanism for facilities to support and guarantee the numerous reform tasks to the greatest extent. In the aspect of security, we should consolidate the basic position of location information and geographical data. On the other hand, the state has clearly defined the supervision standards in improving the management mechanism, the construction of laws and regulations, the optimization of service organization, the cultivation of excellent talents, and the implementation of planning(Chen Chen,2018). At the same time, the guidance and support of various policies, and the gradual improvement of relevant standards and norms. The continuous expansion of service breadth and depth provides a strong guarantee for the long-term sustainable development of the geographic information industry.

\subsection{Development opportunities brought about by} strong demand

In the context of the era of big data, the public's perception of data information and data services is constantly recognized, and the demand for services at different levels of individuals, enterprises and countries is stronger. The real-time online service of electronic map can fully meet the basic needs of individual users for real-time positioning and navigation, order takeaway, travel, and drip taxi. Major shopping malls, McDonald's, Apple and other enterprises mine and analyze big data to meet the needs of its precise location. Similarly, Tencent, Taobao and other electric businesses can accurately recommend more interesting content to users through their data tracking services(Guohui Luo et al, 2019). Obama pointed out that the "big data strategy" will rise to the level of national will(Shanshan Yang,2017) and China's stated that the possession and control of data will become the core assets of competition among countries. The strong demand in various fields puts forward new demands and new market space for the vigorous development of the geographic information industry.

\subsection{Development opportunities brought about by} the development of university courses

At the present stage, the major of artificial intelligence, GIS and surveying and mapping engineering are widely introduced in Chinese colleges, which has cultivated a large number of excellent talents in various fields and provided basic guarantee for the lasting progress of surveying and mapping geographic information. At present, China provides various support and guarantees for talent cultivation, various evaluation systems are continuously refined and improved, professional teachers are further expanded, management models are innovated and optimized, scientific research capabilities are greatly improved, and increases the number of high-level core papers and patents year by year(Yu Liu et al, 2015). The establishment of key scientific research laboratories, the funding support of national related projects, the timely update and optimization of relevant professional software, the emphasis on the cultivation of students' multidisciplinary background, and creating more innovative products that meet the needs of the society. The introduction of relevant professional college courses, and the efforts of scientific research staff, and joint efforts to combine book knowledge with practical applications, to achieve the transformation of technological achievements into actual production, and promote the vigorous development of surveying and mapping geographic information.

\subsection{Development opportunities brought by} thecomplete industrial infrastructure

The development of any discipline and industry is inseparable from the innovation of science and technology, and the support of software and hardware 
facilities. At present, the continuous improvement of China's industrial infrastructure and related technologies has brought unprecedented opportunities for its development. China's beidou-3 system accelerates the construction speed and adds navigation and communication functions on the basis of various traditional services to achieve the goal of global service(Li Yu et al, 2015). The construction of space and aviation remote sensing system will be accelerated, and the earth observation data will be shared by users at home and abroad. The ability to acquire space-ground integrated data will be further improved, the resolution of satellite remote sensing images has been gradually improved, and the display of detailed information has been more accurate and precise. At the same time, the national geographic information database and service platform have been fully built and put into use (Zhenshan Yang et al, 2015 ). The continuous improvement of relevant infrastructure provides basic guarantees and development opportunities for industrial development.

\subsection{Development opportunities brought about by} transformation under reform and innovation

Massive data does not have its own value. Only through data mining and analysis, summarizing the rules, drawing relevant models, investing in research and production applications, numerous data can be added value (Wei Yang et al, 2018). Under the technological innovation brought by big data, the service scope and boundary of the geomatics industry are continuously extended. The initial stage of geographic information mainly serves related fields such as measurement and geography. Now it is combined with various advanced technologies to carry out good cross-border and transformation, constantly creating new development concepts, service ideas and profit models, and providing a more intelligent service level. As a modern new service industry and high-tech industry, surveying and mapping geographic information, in the context of innovative ideas and big data, provides an in-depth understanding of the market and achieves a perfect transformation, providing unprecedented opportunities for its growth and development.

\subsection{Development opportunities brought about by service capitalization}

Capital is a necessary prerequisite for the long-term development of an industrial cycle. Massive data and location information resources have fully attracted the communication industries (China Mobile, China Unicom, telecommunications), e-commerce industries (Tencent, Alibaba), service industries (takeaway order) and many other industries to take the initiative to invest in geomatics information services(Tao Gao, 2017). Establishing good cooperative relations with related industries such as real-time navigation, remote sensing data and spatial data, and injecting new vitality and capital into the development of the industry; on the other hand, the industry is integrated with the laws of market economy development and fully integrated into economic and social development. To maximize the social value of surveying and mapping geographic information services, and make rational planning for the advancement of the industry in the environment of service capitalization.

\section{Challenges of mapping geographic}

information in the era of big data

Opportunities and challenges always complement each other. On the one hand, the development of big data brings many development opportunities for geomatics information. On the other hand, we must also actively respond to the challenges posed by the times. The challenges presented are mainly as follows:

\subsection{Challenges arising from the evaluation mechanism of data quality}

In the era of big data, there are many ways to source data. In addition to traditional Earth observation data and census data of geographical conditions, it also includes Internet data, real-time data, detection data and sensor data( Xingwen Wang et al, 2016). Due to the different data standards of various departments, the data evaluation mechanism is imperfect, and it is often difficult to make a normative and systematic evaluation of the quality of the acquired big data. The poor representativeness of the data will lead to low credibility in the conclusions of the study. For example, in the social data of the 
Internet, it is difficult to reflect the user group of the elderly. The same VGI data mostly records the economically developed areas, ignoring the remote areas. The data will produce the phenomenon of "data blind area" (Xuejian Zhu et al, 2015), resulting in incompleteness of the sample data. The standardized evaluation mechanism of data quality is the guarantee of data processing, data visualization and correct storage of data.

\subsection{The challenges brought by insecurity of data sharing channels}

The era of big data requires the opening and sharing of massive data information. Based on the principle of data confidentiality and intellectual property protection in China, people are questioning the security and confidentiality of data sharing channels. Today, data sharing reduces the security of data while providing convenience. Each user has private information such as multiple APP login accounts, passwords, bank payment account passwords, and location data(Zhengfang Jiang, 2012). If such data cannot be legally protected, there will be a large number of data security risks, at the same time the rights of information providers cannot be guaranteed. At present, the data security management and supervision mode of the Internet platform still needs to be continuously strengthened. In 2019, Ma Huateng, Li Yanhong and others put forward the urgent requirements for the protection and legal utilization of big data at the two sessions(Liguo Xin, 2016). The state should make macro regulation, management and integration of the security and confidentiality of the industry. Only by actively meeting and overcoming the challenges of data sharing can the security of data be guaranteed.

3.3 Challenges brought by the ambiguity of management objects under the survey of the whole people

In the context of big data, the process of geomatics information towards universal mapping for the whole people is accelerated, but this development trend will weaken the accuracy of the supervised objects( Shiyou Sun , 2016) . Under the era of universal surveying and mapping, many data come from the online media, and there will be problems such as the ambiguity of managed objects and the difficulty of supervision. It is difficult to ensure the authenticity, accuracy and reliability of data in the virtual network world. Also need to urgently solve the illegal data with the bad data creation, transmission and management, only keep regulation of data sources and transmission can fully guarantee the security of data. In the context of the times, the large and uncertain user groups should be updated in real time with management concepts, clear management objects and increase management efforts to promote the healthy and rapid progress of the industry.

\subsection{The challenges brought by developed} countries to seize the market

Luo Ruilan(Nengcheng Chen et al, 2015), President of IBM, points out that in the future, data will become a natural resource with core competitiveness for human beings. More and more countries are beginning to realize that the size, activity and analytical capabilities of location information and data owned by a country will occupy a pivotal role in comprehensive national strength. The biggest test to each country for huge amounts of data processing and analysis ability, better meet the needs of the public and society, promote the effective operation of social economy. At present, developed countries are accelerating the pace of occupying the international market and constantly narrowing the upside of China's geographic information industry. At the same time, most of the core practical technology intellectual property rights are still controlled by foreign countries. Compared with the service level and quality of developed countries, there is still a certain gap. Under the stimulus of the developed countries to seize the market, China must attach great importance to guiding and planning the development direction of the industry from a global perspective and improving its core competitiveness.

3.5 Innovation and cross-border environment challenge the improvement of related science and technology

"Science and technology are the primary productive forces". As China's geomatics information 
industry is in the stage of large-scale growth, under the environment of promoting scientific and technological innovation and industrial cross-border, it challenges the updating and improvement of related science and technology in China. At present, although China's Beidou satellite navigation is speeding up construction, the service level of location information and precise positioning in China still has a large room for improvement compared with foreign countries. The scale of industrial development still needs to be further expanded. Therefore, China should improve its independent innovation capability. On the other hand, to show a geometric growth and low density value of huge amounts of data, relevant scientific research personnel should improve their data control capabilities and deepen their in-depth application of various advanced technologies based on existing data resources. The use of a variety of algorithms for data values for "purification", making the industry go to the forefront on the international stage.

4. The work enlightenment of promoting the sustainable development of surveying and mapping geographic information

\subsection{Conform to the law of market development}

The geomatics information industry is developing and growing in the economic market, industry and market belong to mutually complementary and inseparable relationship.Therefore, to a certain extent, we must understand the market more deeply, strengthen the management philosophy and business model of the industry in the market economy, in accordance with the law reform of market economy, establish an effective connection mechanism between demand and service, an increase of the theory, technology, industry and market together, and promote the progress and the sustainable development of geographic information enterprises.

\subsection{Improvement of hardware and software technology}

Realize the combination of data mining, cloud computing processing technology and geographic information processing technology to improve the independent innovation capability and core competitiveness of the geomatics information industry. On the one hand, we will reform the data management system to maximize the cost of information and improve the value of the joint construction and sharing of geographic surveying and mapping data. On the other hand, we will reform and optimize the surveying and mapping technical solutions and business models, build a new working model, improve the work efficiency and emergency ability of each link, and actively develop other types of geographic information resources. The software and hardware technologies are simultaneously improved to steadily enhance the overall strength of surveying and mapping enterprises and promote their all-round development.

\subsection{Strengthen data security protection}

The failure of Google influenza trend prediction and the phenomenon of data leakage and reselling have triggered public concern and reflection on the rational use of big data. In order to protect the security of big data, first reduce the hidden dangers of big data security from data sources, and then strengthen the supervision of the standardization in the process of data processing. In the end, the government level should also control the big data industry and perfect the surveying and mapping geographic information industry data privacy and intellectual property laws and regulations. Big data formulates plans for the development of industry and improve the corresponding standards and supervision mechanism, the country actively guides the healthy development of the industry.

\subsection{Fully and rational use of data to accelerate the trend of universalization}

In the special report, McKinsey pointed out that the rational and effective use of massive data will become the basis for the company's growth and competition(Junchang Hu, 2016). At present, China is changing the traditional concept of data utilization and management, forming the transformation of geospatial data to big data model, strengthening the processing and control of virtualized data, and exploring the potential value behind massive data. On the other hand, accelerate the process of universalization, strengthen the public service capacity of the industry, 
and improve the pace of construction of information-based smart cities. At the same time, it pays attention to the cross-border and transformation of the industry, and derive a variety of emerging industries, such as digital administrators and the drone driver, promotes the progress of surveying and mapping geographic information enterprises.

\section{Conclusion}

In the current era of data and information, the traditional geomatics information service model and management philosophy can not fully meet the requirements of the public. In the era of big data, the competition for geomatics information industry is undoubtedly the looting of massive location information data. Sustainable development provides broad opportunities and severe challenges for the advancement of the geomatics information industry. We need to study the connotation and characteristics of big data in depth, change the development characteristics and concepts with the times, make rational use of big data resources, and carry out technology research and development, innovation and upgrade, take the initiative to cross-border and transform the industry, accelerate the development trend of universalization. So that professional users and ordinary users can become the creators and enjoyrs of the geomatics information industry, and maximize the use of surveying and mapping geographic information data. The value of better serving the public and speeding up urban development.

\section{REFERENCES:}

Chunjun Tan. Analysis of the impact of big data and its work on mapping and mapping geographic information $[\mathrm{J}]$ Resource Information and Engineering, 2017, 3201:128-130.

Bojie Fu. The integrated studies of geography: Coupling of patterns and processes. Acta Geographica Sinica, 2014, 69(8): 1052-1059.

Fuhua Ren . Exploring Big Data and Its Implications for Surveying and Mapping Geographic Information
[J]. Industry , 2015(3): 183-184.

Lili Cui , Zhenggang Wang, Jingde Li . Analysis of the opportunities and challenges faced by surveying and mapping geographic information services in the era of big data [J]. Smart City, 2017, 3 (05): 93.

Lazer D, Kennedy R, King G, et al. The parable of Google Flu: Traps in big data analysis. Science, 2014, 343(6176): 1203-1205.

Rabari C, Storper M. The digital skin of cities: Urban theory and research in the age of the sensored and metered city, ubiquitous computing and big data. Cambridge Journal of Regions Econonomy and Society, 2015, 8(1): 27-42.

Chen Chen. Analysis of Opportunities and Challenges Faced by Surveying and Mapping Geographic Information Services in the Age of Big Data [J]. Western Resources, 2018(05): 148-152.

Guohui Luo, Bin Huang. Studying the Opportunities and Challenges of Surveying and Mapping Geographic Information Services in the Age of Big Data[J]. Ju she,2019,(1):175.

Shasha Yang . Big Data and its impact on mapping and mapping geographic information work [J]. Urban Construction Theoretical Research: Electronic Edition, 2017 (27): 128

Yu Liu , Xi Liu , Song Gao, et al. Social sensing: A new approach to understanding our socio-economic environments. Annals of the Association of American Geographers, 2015, 105(3): 1-19.

Li Yu , Feng Lu ,Hengcai Zhang . Extracting geographic information from web texts: Status and development. Journal of Geo-Information Science, 2015, 17(2): 127-134.

Zhenshan Yang, Ying Long, Douay N. Opportunities and limitations of big data applications to human and 
economic geography: The state of the art. Progress in Geography, 2015, 34(4): 410-417.

Wei Yang, Binhua Tan. Application of Big Data in Surveying and Mapping Geographic Information [J]. Smart City, 2018, 4(12): 61-62

Tao Gan .Opportunities and Challenges Faced by Surveying and Mapping Services in the Information Age [J]. Engineering Technology Research, 2017, (4): $254+256$.

Xingwen Wang, Yue Peng, Jianhua Cao . Discussion on the Transformation and Development of Geographic Information in Surveying and Mapping in the Information Age [J]. Quality Exploration, 2016, 13(4): 66-67.

Xuejian Zhu, Jianqiang Fang, Xuebo Ye . Feasibility study on the construction of provincial mapping and geographic information big data center [J/OL]. Geography Spatial Information, 2015, 13(3): $77-78+10$.
Zhengfang Jiang . Discussion on How to Improve the Management Level of Shanghai Surveying and Mapping Geographic Information[J]. Shanghai Urban Planning, 2012, (4): 98-101.

Liguo Xin . A brief discussion on the opportunities and challenges faced by surveying and mapping geographic information services from the perspective of big data development [J]. Science and Technology outlook, 2016, 12: 306.

Shiyou Sun, Xian Yang, Hongfen Yang. Geographic information service model based on large map [J]. Surveying and Mapping Science, 2016 ( 02) .

Nengcheng Chen, Wang Xiaolei, Xiao Changjiang, et al. Event-driven city information focusing service model and system [J]. Journal of Wuhan University • Information Science Edition, 2015, 40(12): 1633-1638 Junchang $\mathrm{Hu}$. Analysis of the role of mapping and mapping geographic information in the construction of smart cities[J]. Engineering and Technology Research, 2016, (6): 256 